\title{
Correction to: Classical Topics in Complex Function Theory
}

\section{Reinhold Remmert}

\section{Correction to:}

R. Remmert, Classical Topics in Complex Function

Theory, Graduate Texts in Mathematics 172,

https://doi.org/10.1007/978-1-4757-2956-6

This book was inadvertently published without updating the following corrections in Chapters 2, 5, 8, 10, 12, 13, 14, and in the back matter. These are corrected now.

\section{Chapter 2}

p. $47,1.13$ : " $\mathbb{C}^{-”}$ should be " $\mathbb{C}^{-}=\mathbb{C} \backslash(-\infty, 1]$ ".

\section{Chapter 5}

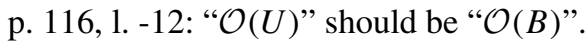

\section{Chapter 8}

p. 171, 1. -6: "Null homologous" should be "null homotopic".

p. $173,1.14$ : " $\mathbb{R} "$ should be “CC".

p. 177,1 . -7: " $G$ to $E$ " should be " $G$ in $E$ ".

p. 178, 1. 12 and 13: "Since square roots are used in the construction of expansions, it is hardly surprising that..." should be "That square roots are used in the construction of expansions is hardly surprising, since...". The sentence should read: "That square roots are used in the construction of expansions is hardly surprising, since $x \mapsto \sqrt{x}$ is a simple expansion of the interval $[0,1)$." 
p. 180, 1. 6: In statement ii), insert "(has an antiderivative)" between "integrable" and "in". The sentence should read "Every function holomorphic in $G$ is integrable (has an antiderivative) in $G . "$

p. 181, 1. -7: "Gedächniss" should be "Gedächtniss".

p. 188, 1. -2: "Groups" should be "domains".

\section{Appendix to Chapter 8}

p. 195 , 1. -1 and -2 : Replace “... that the limit map in 2) is, by 8.2 .5 , the uniquely determined map $G \stackrel{\sim}{\rightarrow} \mathbb{E}$ ” by “... that the limit map in 2) is the map $G \stackrel{\sim}{\rightarrow} \mathbb{E}$, which is uniquely determined by 8.2.5."

p. 196, 1. -5: " $\tau_{2}$ " should be " $\tau_{2}(x)$ ".

\section{Chapter 10}

p. 225, 1.1 (chapter epigraph): Insert "égale" between "jamais" and "ni" and omit the comma. The French sentence should read: "Une fonction entière qui ne devient jamais égale ni à $a$ ni à $b$ est nécessairement une constante."

\section{Chapter 12}

p. 268, 1. 18: "Pole-shifting" should be "pole-pushing".

p. 272, 1. 10 and 1. 16: "Pole-shifting" should be "pole-pushing".

p. 273, 1. 5: "Pole-shifting" should be "pole-pushing".

p. 274, 1. 5: "Pole-shifting" should be "pole-pushing".

p. 277, 1. -6: "Pole-shifting" should be "pole-pushing".

p. 284, 1. 6-7: Insert "of equal length" after " $k$ segments $\left[p_{1}, p_{2}\right],\left[p_{2}, p_{3}\right], \ldots,\left[p_{k}, p_{1}\right]$ ". The first part of the sentence should read: "A closed polygon $\tau=\left[p_{1} p_{2} \ldots p_{k} p_{1}\right]$ composed of $k$ segments $\left[p_{1}, p_{2}\right],\left[p_{2}, p_{3}\right], \ldots,\left[p_{k}, p_{1}\right]$ of equal length is called...".

\section{Chapter 13}

p. 294, 1. -14: "Pole-shifting" should be "pole-pushing".

p. 305, 1. 20: At the end of the line, " $1 \leq n \leq \infty$ " should be " $1 \leq n<\infty$ ".

p. 305,1 . 24: At the beginning of the line, " $D$ " should be “ $\mathbb{C} "$.

\section{Chapter 14}

p. 310, 1. 2: "CC" should be “伊".

p. 312, 1. -13: "Example 13.A.1(4)" should be "13.A.1(3)". That is, "(4)" should be "(3)" and the word "Example" should be omitted.

p. 317, 1. 11: "Basis of $G$ " should be "basis of $H(G)$ ". 


\section{Biographies}

p. 323, 1. 17: "Berl." should be "Verl."

\section{Symbol Index}

p. 329: Add " $\mathbb{N}_{m}, 8$ " between " $\Pi_{v=k}^{\infty}, 4$ " and the next entry.

p. 329: Add " $g_{c}, 176$ " between " $P D(f), 126$ " and "Aut $G, 188$ ".

\section{Name Index}

p. 332, 1. 23: In the entry for Grunsky, "1936" should be "1986".

\section{Subject Index}

Additional page numbers should be inserted in the appropriate numerical order.

p. 337, entry for "admissible expansion": Add "199".

p. 342, entry for "hole": "290" should be "291".

p. 342 , entry for "homology group of a domain": Add " 210 ".

p. 344, entry for "modulus of an annulus": Add "171".

p. 345, entry for "null homologous cycle": Add " 310 ".

p. 346: "Pole-shifting technique" should be "pole-pushing technique".

p. 346: "Pole-shifting theorem" should be "pole-pushing theorem".

p. 348: Add "Stirling's formulas, 58" between the entries "step polygons, Jordan curve theorem for" and "Stirling's series".

p. 348, entry for "Šura-Bura's theorem": Add " 291 ".

p. 349, top of page: Add “Schönflies's, 187" immediately after "Runge's, on rational approximation, 292".

p. 349, entry for "Šura-Bura's": Add "291". 